Dr. Keber bei den Najaden und Dr. Nelson bei den Ascariden behaupteten Eindringens der Spermatozoiden in das Ei. Gießen 1854. - id., Bestätigung des von Dr. Newport bei den Betrachiern und Dr. Barry bei den Kaninchen behaupteten Eindringens der Spermatozoiden in das Ei. Gießen 1854. - id., Historisch-kritische Bemerkungen zu den neuesten Mitteilungen über die erste Entwicklung der Säugetiereier. München 1877. - Bueß-Joël, Gesch. Überblick der Spermatozoenforschung..., Mschr. Gebh. 114, 1942, 70-96, 205-223. - Claparède, Z. wiss. Zool., 9, 1858, 106-128. - Fol, Arch. Sciences phys. et nat. 58, 1877, 439-472. - id., Mém. Soc. phys. et d'hist. nat. de Genève 26,1879,89-250; 251-397. - Haberling, Johannes Müller. Leipzig 1924. - Handwörterbuch der Naturwissenschaften, 2. A., Bd. 3, Jena 1933 (Art. «Ei»). - Hertwig, Morphol. Jahrb. 1, 1876, 347-434. - id., Dokumente z. Gesch. d. Zeugungslehre, Arch. mikr. Anat. 90, 1918, Abt. II. - F.Keber, De spermatozoorum introitu in ovula. Königsberg 1853.-Kölliker, s. bei Bueß-Joël. - Korschelt-Heider, Vergl. Entwicklungsgeschichte der Tiere. Bd. 1, Jena 1936. - Lacaze-Duthiers, Ann. Sciences nat., zool., 7, 1857, 1-51. - Leuckart, Handwörterbuch der Physiol., Bd. 4, Braunschweig 1853, 707-1000. - id., Arch. Anat. und Physiol. (J. Müller) 1855, 90-264. - Malpighi, Opera omnia. Lugd. Bat. 1687 (tom. II, Diss. I.). - Meißner, Z. wiss. Zool. 5, 1854, 207-284. - id., ib., Bd. 6, 1855, 208-266. - id., Verh. Nat. Ges. Basel 1856, H. 3, 374. Müller, Über die Larven und die Metamorphose der Echinodermen. Berlin 1852. - id., Arch. Anat. und Physiol. 1854, 60-68. - (Die Monatsber. d. Berl. Akad. 1851 waren mir für längere Zeit nicht zugänglich.) - Newport, Philos. Tr. 1853, p. II, 233-290. Nordenskiöld, Geschichte der Biologie. Jena 1926. - Prévost-Dumas, Ann. Sciences nat. 1, 1824, 1, 167, 274; 2, 100-129. - Pringsheim, Monatsber. Berl. Akad. Wiss. 1855, 133-164; 1856, 225-237. - Quatrefages, Ann. Sciences nat. zool., 13, 1850, 128-140. Rábl, s. bei Hertwig (1918). - Robin, Journ. physiol. 5, 1862, 67-109. - Spallanzani, s. bei Bueß-Joël. - Thomson, Cyclop. of Anat. and Physiol. 5, 1859, 98-137. - Warneck, Bull. Soc. Imp. des Naturalistes de Moscou, 23, 1850, 90-194.

\title{
La condromatosi articolare nell' opera di Morgagni
}

«... die alte, zuletzt dogmatisch gewordene Medizin ihre Freiheit wiedergewonnen hat und zu der neuen naturwissenschaftlichen Medizin geworden ist. Dieser denkwürdige Wechsel ist durch lange Kämpfe eingeleitet worden, welche zum großen Theile auf dem Boden Italiens ausgefochten worden sind, wenngleich an deren siegreicher Beendigung auch andere Nationen betheiligt waren.»

Virchow (50)

Si può senza dubbio affermare che $i$ due pilastri fondamentali del moderno sapere anatomo-patologico - accanto ad altri solidissimi, come quelli costruiti da v. Rokitansky e da Cruveilhier - sono il: «De sedibus 
et causis morborum per anatomen indagatis (1761) di Morgagni $\left({ }^{33}\right)^{1} \mathrm{e}$ la Cellularpathologie (1858) di Virchow ${ }^{48}$ ). Opere innovatrici in modo ugualmente profondo, nonostante che la prima, di indole essenzialmente anatomo-clinica, sembri maggiormente ricollegarsi, non foss'altro che per la lingua usata, alla precedente tradizione medica; mentre la seconda, a carattere eminentemente microscopico, sembra presentare, anche per l'uso della lingua nazionale, impostosi nella letteratura scientifica del XIX ${ }^{0}$ secolo, un carattere più schiettamente rivoluzionario.

L'incessante progredire della scienza, proteso verso l'avvenire, ci costringe troppo spesso a distogliere lo sguardo dai grandi del passato; tuttavia la ricerca - nell'elegante e forbito latino di quella miniera casistica che è il De Sedibus - del caso analogo a quello dell'autopsia appena finita può costituire per un patologo un otium piacevole e capace di ritemprarlo dal freddo del tavolo anatomico.

Ci è così capitato di imbatterci nella descrizione di un caso, che, a nostro avviso, deve essere interpretato come una condromatosi articolare. Esso è esposto negli artt. 14-15 della Epistola anatomico-medica LVII e nell'art. 13 della Epistola LXIX.

Si tratta di una donna anziana, madre di parecchi figli (anus, complurium liberorum pariens), la quale circa tre mesi prima della morte fu colpita da un insulto apoplettico (ante menses circiter tres apoplexia correpta fuerat), che ebbe come conseguenza una emiparesi motoria destra (resolutio exceperat artus utriusque dexteri sic tamen, ut salva sentiendi facultate, moveri nonnihil possent). La morte avvenne nel dicembre del 1746 (e vita excesserat post initium Decembris A. 1746) e l'autopsia, che durò parecchi giorni, dovette essere particolarmente ricca di reperti, perchè il Morgagni dichiara di riportare soltanto quelli attinenti al soggetto dell'epistola (De multis quae pluribus diebus secando, in ejus corpore animadverti, haec sunt potissimum quae ad huc attinent). Sorvoliamo sopra i reperti extra-articolari, in particolare sopra quelli cerebrali, sui quali il Morgagni si diffonde, per soffermarci sulla descrizione del ginocchio sinistro.

Avedo sezionato questa giuntura a scopo di dimostrazione (ad interiora

1 Il De Sedibus ebbe i suoi più insigni precursori nel De abditìs nonnullis ac mirandis morborum et sanationum causis (1507) del fiorentino Antonio Benivieni e nel Sepulchretum (1679) (4) del ginevrino Théophile Bonet, sulla quale ultima opera il Morgagni si attarda particolarmente nella prefazione del De sedibus. 
vincula, cartilagines et glandulas frequentissimis, qui et superiora omnia viderant, Spectatoribus demonstranda), l'attenzione del Morgagni fu particolarmente colpita dall'assenza di liquido sinoviale (cum mucilaginis nihil quidquam conspicerem), il qual fatto lo indusse a condurre un esame particolarmente scrupoloso (attentius inspectare coepi omnia).

La superfice dei componenti il cavo articolare si presentava abbastanza liscia (satis erant lubrica). La cartilagine rotulea (cartilagineam rotulae crustam), nella sua porzione corrispondente al condilo femorale esterno (qua parte externo femoris condylo respondebat), era come usurata (quasi attritam), presentando dei piccoli e leggeri solchi paralleli, come se fosse stata più volte sottoposta all'azione di una punta di coltello scorrente dall'alto al basso (et levibus parallelis sulculis haud aliter exaratam, quam si quis scalpri aciem, ab summo ad imum saepius ducendo, appressisset). La corrispondente cartilagine di incrostazione del condilo femorale esterno (crustam autem condyli quem modo dicebam, qua eidem rotulae parti obvertebatur) era così assottigliata da apparire alquanto livida a causa del trasparire dell'osso sottostante (adeo extenuatam, ut lividula, ob transpectum per ipsam os, appareret).

Ma il reperto più interessante (prae his autem multo observatione digniores) era costituito dalla presenza di numerosi globuli (globuli plurimi), di cui i cinque più grandi raggiungevano le dimensioni di un acino d'uva di media taglia (quorum maximi quinque pares erant ferme omnes mediocribus uvarum acinis), mentre i restanti, in un numero superiore ai venti, erano di dimensioni chi più chi meno inferiori (reliqui, plures viginti, alii aliquanto, alii multo illis minores). ${ }^{2} \mathrm{Di}$ colore bianco (albi erant omnes) ed a superfice liscia (et laevi superficie), tutti questi globuli erano separati gli uni dagli altri (omnesque inter se disjuncti) e aggettavano all'interno del cavo articolare (intra articuli cavum prominebant).

In parte peduncolati (exigua sui parte adhaerentes), in parte sessili (continuati), i più piccoli di essi aderivano ai lati inferiori della capsula, formando alcuni una lunga fila (minores quidem capsulari ligamento circa huius latera inferiora sic ut aliqua in oblongam seriem disponerentur).

I più grandi invece-così suona, letteralmente tradotto, il testo del

$2 \ll \ldots$ stecknadelkopf- bis erbsengroßer knorpeliger Geschwïlste ...». Reichel (44) (p. 720). 
Morgagni-si penducolavano o si continuavano con la cartilagine di incrostazione o piuttosto con le estremità di alcune ghiandole muccose, che coprivano strettamente la cartilagine di incrostazione in alcuni recessi delle ossa nella loro porzione intra-articolare (exigua sui parte adhaerentes, imo continuati ... majores autem cartilagineae crustae, seu potius fimbriae glandulae alicujus mucilaginosae, cartilagineam crustam arcte operientis in recessibus quibusdam ossium ad cavum articuli pertinentium).

Per interpretate correttamente questo brano, dobbiamo anzittutto ricordare che era opinione corrente al tempo del Morgagni, radicatasi in seguito alle ricerche di Havers (1691) (19), che le frange ed i villi sinoviali fossero ghiandole muccose, deputate alla secrezione della sinovia: è merito di Bichat $\left({ }^{3}\right)$, il fondatore dell'anatomia generale, di aver dimostrato nel 1799 che la sinoviale è una membrana sierosa anzichè muccosa e che i rilievi apprezzabili alla sua superfice interna non sono ghiandole muccose, bensì semplici ispessimenti e plicature di questa membrana.

Tenendo presente questo fatto, il brano del Morgagni può essere interpretato nel senso che i globuli più grossi facevano corpo con le frange e villosità della sinoviale, in tal guisa proliferata da coprire per un certo tratto la cartilagine di incrostazione dei capi articolari. Nella moderna letteratura sulla condromatosi articolare esistono parecchie osservazioni, in cui è chiaramente descritta una proliferazione, a tipo di panno, della sinoviale al di sopra delle porzioni marginali delle cartilagini di incrostazione, accompagnata dalla produzione di noduli cartilaginei da parte della sinoviale in tal guisa proliferata. Tale processo è chiaramente descritto, tra gli altri, da Reichel $\left({ }^{44}\right)^{3}$, Lexer $\left({ }^{31}\right)^{4}$, Troell $\left({ }^{47}\right)^{5}$ nell'articolazione del ginocchio, da Hagemann $\left({ }^{16}\right)^{6}$ in quella della spalla e da quest'ultimo molto bene rappresentata nella fig. 3 del suo lavoro.

Alcuni globuli contenevano un nucleo osseo sotto una scorza cartila-

3 «Nur um wenige Millimeter weit griffen die knorpeligen Zottenwucherungen auf den Rand der Gelenkknorpel - wie beim tuberkulösen Pannus - über», Reichel (44) (p. 721).

4 «Der Rand der Gelenkknorpel war nur wenige Zentimeter weit von den knorpeligen Zottenwucherungen befallen», Lexer (31) (p. 312).

5 «Von den Rändern der Condylen kriechen die Kapselveränderungen mehr oder weniger weit über die Gelenkflächen hin», Tröll (47) (p.682).

${ }_{6}^{6}$ «Auch auf den dem Rande benachbarten Partien der Knorpeloberfläche des Humeruskopf ... finden sich die gleichen Knötchen. Der knorplige Überzug der Gelenkpfanne ist dagegen frei, ihr Rand aber wieder dicht mit Knötchen besetzt». «... die Knötchen, welche im Bereiche der Gelenkknorpel sitzen, denselben nur überziehen, nicht in denselben eindringen», Hagemann (16) (p. 1244). 
ginea (alii sub cartilagineo cortice osseum nucleum continebant), altri invece erano completamente ossei (alii omnino erant ossei): costatazione questa ripetuta in seguito (non omnia cartilagineo cortice induta, sed plura invenerim omnino ossea).

Purtroppo non potè essere eseguito l'esame del ginocchio sinistro: infatti, desiderando il Morgagni constatare l'eventuale presenza dei globuli in questa giuntura, onde poter rilevare qualche reperto che meglio ne illustrasse il meccanismo genetico (cum in altero quoque genu vellem quaerere, si forte inessent, meliusque originem ostenderent suam), provò la delusione, anzi il dolore (aegre tuli), di sapere che il pezzo anatomico era già stato sepolto (jam cum plerisque aliis partibus fuisse humatum). E questa una delusione piuttosto di uso corrente per gli anatomo-patologi di ogni tempo e di ogni paese!

Commentando in seguito il reperto, l'Autore dice che, qualsisia l'origine dei globuli (unde unde extiterint), certamente la loro presenza deve secondariamente ripercuotersi in modo dannoso sullo stato anatomico e funzionale della giuntura. Precisamente egli enumera le due seguenti possibilità:

1) sia che essi fossero nati dalle ghiandole muccose (sive ex mucilaginosis excreverant glandulis) - e qui ricordiamo che il Morgagni aderiva all' opinione di Havers che le frange ed i villi sinoviali fossero ghiandole muccose destinate alla secrezione della sinovia - avrebbero potuto nuocere alla giuntura (nocere articulo potuisse) diminuendo la secrezione della sinovia (secretionem mucilaginis imminuendo)

2) sia che diversa fosse la loro origine (sive aliunde erant) - anche se finora non erano stati molto dannosi (si hactenus haud multum nocuerant), il che del resto non si potè stabilire con sicurezza (neque enim id scire pro certo licuit) - ugualmente avrebbero potuto, aumentando di volume, nuocere alla giuntura (plurimum, augescendo, fuisse nocituros). $\mathrm{Si}$ sarebbe infatti molto vicini alla verità (non is loqueretur a veri similitudine omnino abhorrentia), affermando (si quis forte diceret) che uno di questi globuli, sia pur piccolissimo (ex iis osseis globulis minimum quempiam), distaccatosi (e sua avulsum sede) durante i movimenti del ginocchio (genu se agitante) ed interpostosi tra il condilo femorale e la rotula (atque inter condylum et rotulam interjectum), abbia provocato le descritte lesioni delle cartilagini di incrostazione (utriusque cartilagini ea vitia attulisse quae indicata sunt). Il qual concetto, espresso in linguaggio moderno, significa che le lesioni, notate alle superfici articolari della 
rotula e del femore, non sono altro che fenomeni di artronosi deformante secondaria: sono cioè manifestazioni consecutive al danno che la presenza di corpi stranieri induce sullo stato anatomico e funzionale della cartilagine di incrostazione e quindi dell'intero capo articolare (teoria funzionale dell'artronosi deformante, Pommer $\left({ }^{4142}\right)$. Che tale sia l'interpretazione del Morgagni lo deduciamo anche dall'accostamento, da lui fatto, con un probabile caso iniziale di artronosi deformante, apparentemente primaria o essenziale (quanquam memini, cum insequenti anno ibidem nescio cujus hominis genu perlustrarem, crustam ejusdem condyli cartilagineam eadem illa parte qua rotulam tangit, nonnihil quasi corrosam invenisse non majori spatio quam quod unguis digiti minimi operire potuisset, exiguisque aliis in ea vicinia spatiolis; tametsi nec in rotula quidquam vitii, neque alibi in toto articulo appareret; nedum globuli extarent nulli).

L'Autore passa poi all'esame critico comparativo del suo caso con reperti analoghi osservati da Haller e da Reimar. Come dice esplicitamente il Morgagni, si tratti di reperti simili ma non analoghi: istiusmodi in genu morbi qui mentionem fecerit, neminem videor legisse; ed in seguito: haec igitur, quae non repetendi sed comparandi causa a me dicta sunt. Purtroppo, nonostante tutte le ricerche fatte, non ci è stata possibile la diretta consultazione delle opere dei due suddetti Autori e pertanto il seguente commento delle loro osservazioni è basato esclusivamente su quanto di esse ci è riferito dal Morgagni. -

Osservazione di Haller (Observatio Illustris Halleri). ${ }^{7}$ Si tratta di una donna molto vecchia (in decrepita muliere), affetta da grave aterosclerosi (cuius arteriae plures et aliquae cordis valvulae osseis squamis, aut harum inchoamentis non carebant), nella quale l'esame delle articolazioni temporo-mandibolari (dum inferioris maxillae cum ossibus temporum articulos introspiceret) mostrò che, mentre l'una di esse era indenne (omnia secundum naturam), l'altra invece presentava una scomparsa delle cartilagini di incrostazione (nudatum crusta cartilaginea) ed una grave usura

7 L'osservazione è riportata in un opera di Haller, della quale il Morgagni dà la seguente citazione bibliogafica: Progr. de indurat. corp. hum. partib. § 5. Tale opera nơn è citata nell'elenco delle opere di Haller riportato nel Denkschrift (18), che la città di Berna ha dedicato al suo grande figlio nel 1877, in occasione del $100^{\circ}$ anniversario della sua morte. Però nel Catalogus omnium operum Alberti de Haller, riportato alla fine degli Opuscula pathologica (17), si trova la seguente citazione: 84 . De induratis partibus corporis humani, Gotting. 1753. 4. 
e perforazione del menisco (et meniscum pene dimidium consumatum et perforatum). Lo sfregamento articolare - e queste sono parole di Haller - aveva provocato queste lesioni della cartilagine d'incrostazione, trasformandola in una ventina di frammenti di forma globulare (crustam quam tritus deleverat, idem tritus in globulos fere viginti mutaverat, semiosseos et in capsulae cavea libere collectos): reperto questo che sorprese non poco lo stesso osservatore, talchè fu da questi definito come un mirum spectaculum. Haller non deve essersi molto diffuso a proposito di questo caso, perchè Morgagni ci assicura che, se l'Autore avesse riportato maggiori particolari, questi ci sarebbero stati riferiti onde meglio noi potessimo paragonare le osservazioni dei due grandi maestri (plura, si plura addidisset, describerem tibi ut omnia cum mea posses Observatione conferre): ad ogni modo anche di questo caso mancano notizie precise sulla sintomatologia clinica (verum nec ipsi pro certo resciscere licuit, ut videtur, quid inde, quantumve incommodi cepisset foemina).

Siamo contrari ad interpretare il caso di Haller come una condromatosi articolare. A parte la considerazione che in 101 casi di questa malattia, raccolti da Chiari $\left({ }^{7}\right)$ nella moderna letteratura, non figura nemmeno una volta come sede l'articolazione temporo-mandibolare, va notato che nella descrizione non è fatto cenne di corpi sessili o peduncolati. Ci associamo pertanto alla opinione dello stesso Haller che i corpuscoli liberi nel cavo articolare siano derivati da usura delle cartilagini di incrostazione. La presenza di una grave aterosclerosi cardio-aortica costituisce poi un dato, che, per quanto di valore relativo, parla in favore dell'artronosi deformante, malattia che da qualche autore, ed anche da noi, viene con. siderata come un processo aterosclerotico a carico delle giunture.

Il distacco dai capi articolari di particelle, più o meno grandi, di cartilagine e di incrostazione è stato infatti osservato in artropatie di vario tipo, ed in particolare nella artronosi deformante (Broca $\left(^{6}\right)$, Pommer $\left({ }^{41}{ }^{42}\right)$, Lang $\left({ }^{28}{ }^{29}\right)$, nella artropatia tabica (Moritz $\left({ }^{34}\right)$, nell'artropatia emofilica (Freund $\left({ }^{11}\right)$. Il destino di tali particelle è alquanto vario: così Pommer nell'artronosi deformante e Moritz nell'artropatia tabica ne descrissero l'incistamento (Detrituscysten di Pommer). Nell'artronosi deformante poi esse possono arrivare, per la via dei capillari venosi e delle vie linfatiche perimielari o perivascolari, negli spazi midollari subcondrali, dove possono proliferare, trasformandosi in noduli cartilaginei (Knorpelknötchen di Pommer e Lang). Nell'artropatia emofilica Freund notò l'innesto di tali particelle nella membrana sinoviale, seguito da una proli- 
ferazione reattiva del tessuto ospitante: reperto analogo fu da noi rilevato in ginocchia affette da artronosi deformante.

Osservazioni di Reimar ${ }^{8}$. - Reimar descrisse nella sua tesi di dottorato di aver visto, inteso e letto, durante il periodo della sua vita di studente passato in Inghilterra, parecchi casi di corpi liberi intraarticolari del ginocchio. Questi erano allora piuttosto ignorati dalla letteratura medica del continente, mentre erano di osservazione alquanto corrente in Inghilterra, tanto da essere chiamati volgarmente sorci del ginocchio (In Anglia, ubi rarus adeo apud nos morbus, non infrequens est, ut vulgo etiam vocent mures in genu, plura ille vidit, audivitve et legit ejusdem exempla).

Sorvoliamo sulla loro sintomatologia clinica, descritta da Reimar e riportata da Morgagni, dato che non vi è il minimo dubbio che nell'opera di Reimar non sia riportata la descrizione di un caso di condromatosi articolare. Si tratta semplicemente di corpi liberi intra-articolari, ancor oggi chiamati sorci delle giunture, quali si possono notare nell'artronosi deformante per distacco di cercini marginali, nella osteocondrite dissecante $^{9}$, forse nei traumatismi, etc. Del resto lo stesso Reimar sembra propendere per questa interpretazione (De corpusculorum autem illorum origine, etsi duo exempla profert, unde videri possit, frustula esse quae vi illata ab ossium extremis discerpta fuerint), benchè egli si riservi un giudizio definitivo dopo l'esame di altri casi (nihil definiri ab se posse, prudenter fatetur ante ulterius exemplorum complurium examen). Va notato però che al lume delle conoscenze odierne non hanno valore contro la loro genesi mediante distacco dai capi articolari le considerazioni fatte da Reimar sul fatto che i corpi liberi sono totalmente rivestiti da cartilagine e che non sempre la loro insorgenza è preceduta da traumatismo (cum undique cartilagine aequali vestita spectentur, nec morbum semper vis extrinsecus illata praecesserit).

Sia di passaggio notato, a testimoniare la precisione bibliografica del Morgagni, il rimprovero da questi, sia pure gentilmente, rivolto a Reimar

8 Reimar Johann Albrecht Heinrich (1729-1814), figlio del noto filosofo e teologo luterano Hermann Samuel, fu professore di fisica e storia naturale ad Amburgo, sua città natale, Studiò nel 1752 a Gottinga - dove Haller insegnò dal 1736 al 1753 [Voss (51), Gruber (15)] - e negli anni seguenti a Leida, Edimburgo e Londra. Nel 1756 ritornò a Leida, dove nel 1757 si addottorò con la dissertazione: De tumore ligamentorum circa articulos, fungo articulorum dicto. La citazione bibliografica riportata dal Morgagni è la seguente: De Fungo Articulor. §54 et seqq.

9 Descritta nel 1888 da Franz König (1832-1910) (25), professore di chirurgia a Rostock, Gottinga (1875-1895) e Berlino; noto studioso della tubercolosi osteo-articolare. 
per non aver fatto cenno dell'osservazione di Haller, il quale gli era stato maestro (Illustris ejus Praeceptor Hallerus) nel periodo da lui passato all'università di Gottinga (fugit enim, ut fit, Eruditissimum Reimarum, negantem, in alio ac genu articulo sibi nota esse huius morbi exempla; fugit, inquam, Halleri Observatio, qui morbum hunc in articulo maxillae ostendit, ostenditque simul ibi cartilagines ex parte absumptas).

Ricordiamo infine che, secondo gli Autori francesi (Lannec (27), Cruveilhier $\left({ }^{8}\right)$, Malgaigne $\left({ }^{40}\right)$, Moullonguet $\left({ }^{35}\right)$ spetta ad Ambroise Parè, il padre della chirurgia francese, il merito di avere compiuto per primo, nel 1558, l'estrazione di un corpo libero intra-articolare. ${ }^{10}$

Ritornando ora al caso del Morgagni, ci sembra che esso possa essere unicamente interpretato come una condromatosi articolare: data la presenza di qualche nodulo puramente osseo (alii omnino erant ossei) e volendo seguire la nomenclatura di Kienböck $\left({ }^{23}\right)$, si potrebbe anche parlare di osteocondromatosi articolare. L'artropatia tabica - processo in cui si notano con particolare frequenza produzioni osteo-cartilaginee a carico della sinoviale, come documentò anche microscopicamente Moritz ${ }^{(34)}$ - può essere esclusa per la scarsissima compromissione deformante dei capi articolari.

L'età ed il sesso depongono contro la diagnosi di condromatosi, dato che dalla statistica di Chiari $\left({ }^{7}\right)$ risulta in modo indubbio una netta prevalenze per il sesso maschile e per l'età media: però la moderna letteratura non manca di casi a carico di donne anziane. Ci limitiamo a citare il caso $3^{0}$ di Kopp $\left({ }^{26}\right)$ ( 9 di 77 anni), il caso di Knoblich $\left({ }^{24}\right)$ ( 9 di 76 anni), due casi di Panner $\left({ }^{39}\right)$ riferentisi rispettivamente alle figg. $7-8 \mathrm{e}$ 9-10 del suo lavoro ( + di 70 anni e $q$ di 65 anni), il caso di Jones, citato da Chiari ( $\left.{ }^{7}\right)$, ( 9 di 66 anni).

La sede è invece classicamente tipica: l'articolazione del ginocchio è infatti colpita, nella statistica di Chiari $\left({ }^{7}\right), 46$ volte su 101 casi, essendo seguita a grande distanza dal gomito (29 casi).

Inoltre tutti i caratteri dei noduli (forma, struttura, grandezza, sede, etc.) sono dati che ci portano alla diagnosi di condromatosi.

10 «L'an mil cinq cents cinquante-huit, fus appelé de Jean Bourlier, maistre tailleur d'habits, demeurant rue sainct Honoré, pour luy ouurir vne aposteme aqueuse qu'il auoit au genoüil: en laquelle trouuay vne pierre de la grosseur d'vne amende, fort blanche, dure, et polie, et guerit, et encores est à present viuant», Paré (40) (p. 32). 
Il Morgagni ha poi espresso - in modo ora più, ora meno chiaro e quasi sottaciuto - alcuni concetti, oggidí generalmente ammessi, sulla genesi formale delle lesioni, e precisamente:

1) i noduli osteo-cartilaginei non originano mediante distacco dai capi articolari di frammenti osteo-cartilaginei o di particelle di cartilagine di incrostazione (quarum - e qui il Morgagni si riferisce alle cartilagini di incrostazione - et ego nonnullas tunc vidi sulcatas, extenuatasque, nec tamen quantum ad multo pauciora quam ego invenerim, efficienda corpuscula satis esse potuisset; erant enim non viginti, sicuti in Halleri Observatione, sed plura, et in iis non deerant quinque ea magnitudine, ut mediocres fere aequarent uvarum acinos: erantque omnia quae uterque invenimus, globulorum forma). Tale interpretazione del Morgagni è stata sottolineata in modo molto netto da Lannec $\left({ }^{27}\right),{ }^{11}$ il quale era sostenitore della genesi estrinseca dei corpi endoarticolari. ${ }^{12}$

2) i noduli pigliano origine nella capsula e nelle frange e villosità sinoviali (exigua sui parte adhaerentes, imo continuati, minores quidem capsulari ligamento ... majores autem cartilagineae crustae, seu potius fimbriae glandulae alicujus mucilaginosae; ed in seguito: sed omnia cartilaginibus, vel potius mucilaginosis glandulis continuata deprehendi). Il Morgagni qui afferma in modo esplicito soltanto un rapporto spaziale e non genetico tra noduli e sinoviale; però il fatto che egli escluda l'origine dei noduli per distacco dai capi articolari e ne noti la continuità con la capsula ed $i$

11 «On a été jusqu'à présent fort incertain sur la manière dont ces corps étrangers se développent ainsi, au milieu des articulations. Haller et Reimar ont cru qu'ils étaient formés de fragments détachés des cartilagess articulaires. Morgagni a réfuté leur opinion avec beaucoup de sagacité, en remarquant que les érosions des surfaces articulaires, qui coïncident assez souvent avec l'existence des corps cartilagineux et osseux, sont trop peu considérables pour qu'on puisse croire qu'elles soient dues à la séparation de ces corps. Il eût pu ajouter que plusieurs corps cartilagineux sont exactement sphériques et d'un diamètre très supérieur à l'épaisseur des cartilages articulaires.» Laennec (27) (p. 125). Va però notato che l'ultima frase di Lannec è del tutto superflua, dato che Morgagni piglia risolutamente in considerazione sia la forma sferica (globulorum forma), sia le dimensioni (quinque ea magnitudine, ut mediocres fere aequarent uvarum acinos).

12 «Il me paraît très-probable qu'ils se développent à l'extérieur des articulations, et qu'ils is'enfoncent peu-à-peu dans leur intérieur, enveloppés par un prolongement des membranes synoviales. A mesure qu'ils pénètrent dans l'articulation, ce prolongement se resserre et s'oblitère derrière eux, et forme les expansions membraneuses rougeâtres ou ligamenteuses observées par divers auteurs, jusqu'à ce qu'enfin, il se rompe dans quelque mouvement ou par une sorte d'usure, et le corps cartilagineux se trouve libre dans la cavité articulaire», Lannec (27) (p. 127). 
villi sinoviali ci può far supporre che egli non fosse lontano dall'ammetterne la genesi in sede sinoviale. A tal proposito va notato che la metaplasia cartilaginea ed ostea-cartilaginea della sinoviale venne, nello scorso secolo, presunta da Bichat $\left({ }^{3}\right)^{13}$ ed affermata da Rokitansky $\left({ }^{45}\right)^{14}$ e da Virchow $\left({ }^{49}\right)$, il quale parlò di Synovialknorpel. Reichel $\left({ }^{44}\right)^{15}$, in base allo studio microscopico del suo caso eseguito dal patologo Nauwerck, la dimostrò in modo sicuro. Cruveilhier, nell'Essai $\left({ }^{8}\right)$, dice che il caso di Morgagni sembra confermare l'opinione di Bichat, ${ }^{16}$ ed in seguito, nella

13 «On connoît les productions cartilagineuses mobiles et souvent libres dans les articulations. Viennent-elles de l'ossification d'une portion de la synoviale? Je le présume; car souvent on les a vues tenir au cartilage par des expansions membraneuses. J'ai observé, l'an passé, sur un cadavre la portion de synoviale allant du paquet graisseux qui est derrière la rotule, à l'enfoncement qui sépare les condyles du fémur, presque toute cartilagineuse. Si pendant la vie elle se fût détachée par l'effet des mouvemens, cela auroit formé un de ces cartilages mobiles et libres. Au reste, comme je n'ai que ce fait qui me soit propre sur ce point, je ne puis qu'offrir des conjectures, d'autant plus qu'on sait que la synoviale et les membranes séreuses sont de même nature, et que cependant ces dernières ne deviennet presque jamais cartilagineuses. Au reste, ces sortes de productions suivent absolument la marche ordinaire de l'ossification. D'abord cartilagineuses et sans vaisseaux sanguins, elles présentent bientôt, pour peu qu'elles soient anciennes, un centre rouge, puis osseux, qui s'étend du centre à la circonférence, et qui finit quelquefois par envahir tout le cartilage; en sorte que ce sont de véritables os. Cette dernière circonstance est cependant assez rare. L'état où on a trouvé le plus communément ces productions, est celui où elles sont osseuses au milieu, et cartilagineuses à la circonférence. J'en ai rencontré une dans l'articulation du pisiforme avec le pyramidal, qui avoit le volume de la tête d'une grosse épingle, et qui, dans toute son épaisseur, étoit plus dure que l'ivoire.» Bichat (3) (pp. 143-144).

14 «Die Häute können stark wuchern und im Innern bilden: I. Bindegewebe, 2. Fettgewebe, 3. Knorpel- und Knochensubstanz. - Zu 3. Es sind zunächst kleine, rundliche Massen, zuweilen auf einem Stiel, sie werden später größer und adaptieren ihre Form der Umgebung; sie sind eben oder flach konvex oder sogar konkav, werden multipel und artikulieren manchmal miteinander mit Bildung mehrerer Facetten.» «Die Massen werden zuweilen lose und stellen dann eine besondere Form freier Körper dar.» Rokitansky (45), cité par Kienböck (23) (pp. 2-3).

15 «Der Proceß beginnt mit einer Wucherung und Vergrößerung der Synovialzotten. Schon sehr früh treten in ihren Enden Knorpelzellen auf ...», Reichel (44) (p. 722).

16 «Dans une observation rapportée par Morgagni, les portions de cartilage qui manquoient n'auraient pu suffir à former les corps étrangers. Sont-ils le produit d'une végétation particulière de la membrane synoviale, végétation qui, d'abord celluleuse, se détruiroit, se détacheroit et deviendroit libre dans la cavité articulaire? C'est l'opinion de Bichat, que semblent confirmer quelques observations de Monro et de Morgagni», Cruveilhier (8) (p. 103). Va però notato che Monro considerò il corpo peduncolato, da lui trovato nell'arti- 
Anatomie pathologique $\left({ }^{9}\right)$, ammette, accanto ad altri meccanismi di produzione, lo sviluppo dei corpi stranieri nello spessore delle frange sinoviali.

3) i noduli condromatosi aderenti alla sinoviale, possono distaccarsi e dar così luogo alla formazione di corpi liberi endoarticolari (non difficile fieri posse intellexi, ut in genu agitationibus inde aliqua avellerentur).

4) le lesioni dei capi articolari a tipo di artronosi deformante sono manifestazioni secondarie alla presenza dei noduli condromatosi (ex iis osseis globulis minimum quempiam, genu se agitante, e sua avulsum sede, atque inter condylum et rotulam interjectum, utriusque cartilagini ea vitia attulisse quae indicata sunt).

L' esame critico del caso ci porta quindi a concludere che il Morgagni ha realmente descritto un caso di condromatosi articolare e che ne ha rilevato acutamente, sia pure esprimendole qualche volta in modo non molto chiaro, alcune delle peculiarità, che conferiscono alla malattia la sua essenza e che ne spiegano la genesi formale quale è attualmente concepita.

La descrizione del Morgagni, benchè citata o commentata da parecchi Autori, di cui alcuni chiarissimi, dello scorso secolo Laennec ( $\left.{ }^{27}\right)$, Cruveilhier $\left({ }^{8}{ }^{9}\right)$, Bonnet $\left({ }^{5}\right)$, Virchow $\left({ }^{49}\right)$, è poi caduta in dimenticanza.

In quel secolo furono in seguito descritti, per lo più senza connessione tra loro, altri casi: tra essi ricordo quelli di Humphry $\left({ }^{21}{ }^{22}\right)$ e di Berry $\left({ }^{1}\right)$, il quale ultimo estrasse dalla giuntura malata 1047 corpi liberi cartilaginei. Stando a quanto è riportato da Kienböck $\left({ }^{23}\right)$, anche Rokitansky $\left({ }^{45}\right)$ deve avere probabilmente osservato la malattia. Virchow $\left({ }^{49}\right)$ osservò in un ginocchio 60 corpi liberi ed un certo numero di corpi sessili. Il caso di Cruveilhier $\left({ }^{9}\right)$, mirabilmente tramandato dalla fig. 1 , planche 6 , livraison IX del suo classico atlante è caratterizzato dalla presenza nella giuntura del gomito di una ventina di corpuscoli ossei, ${ }^{17}$ sembra piuttosto rientrare nel quadro della osteomatosi articolare di Kienböck ${ }^{(23)}$.

colazione del ginocchio di una donna di 40 anni, come un frammento di cartilagine distaccato ed in seguito ossificato.

17 «La figure I représente une articulation du coude, qui est remplie de corps étrangers articulaires, les uns libres, les autres adhérens à la synoviale par un pédicule extrêmement grêle. La plupart sont contenus dans des cellules ou loges fibreuses à ouverture plus étroite que le fond. Tous sont osseux; leur surface est tuberculeuse: aucun d'eux ne 
Però tali descrizioni non riuscirono a dare alla malattia dignità nosografica propria. Il merito di aver fatto ciò spetta invece a Reichel $\left({ }^{44}\right)^{18}$, il quale, il 18 aprile 1900 , a Berlino, comunicò al $29^{\circ}$ congresso della società tedesca di chirurgia un caso tipico (ginocchio sin. di un uomo di 36 anni), stabalì le caratteristiche fondamentali del quadro morboso, ne interpretò correttamente la genesi formale e battezzò la malattia col nome oggi generalmente usato: la denominazione di condromatosi articolare e quella di malattia di Reichel sono divenuti sinonimi.

Siamo a conoscenza dell'affermazione di Puccinotti $\left({ }^{43}\right)$ che, se il Morgagni avesse dato il suo nome ad ogni particolarità anatomica da lui scoperta, circa un terzo delle parti del corpo umano porterebbe il suo nome: riteniamo tuttavia giusto di proporre che al nome di Reichel, a cui spetta il merito di avere introdotto nella letteratura moderna l'entità nosografica della condromatosi articolare, sia unito il nome di Morgagni, che, per quanto è a nostra conoscenza, ne osservò il primo caso nel 1746 e lo descrisse così accuratamente nel 1761 da precorrere, in alcuni punti, la moderna concezione di Reichel..$^{19}$ Pertanto proponiamo di denominare la condromatosi articolare malattia di Morgagni-Reichel.

présente le moindre vestige de cartilage. Les surfaces articulaires sont rayées dans le sens des mouvements; elles sont déformées: la synoviale est épaissie, mais sans rougeur. Cette figure offre aussi, indépendammẹnt des corps étrangers, des rayures sur la troklée humérale TR et sur la grande cavité sygmoïde CRS et des végétations osseuses VO.»Cruveilhier (9) (pp. 13-14).

La fig. 2 della stessa planche 6 raffigura invece un ginocchio aperto, la cui rotula e femore sono affetti da grave artronosi deformante. Nella giuntura sono presenti 4 corpuscoli, in parte sessili, in parte penducolati, il cui colorito corrisponde a quello della cartilagine (nel testo non è fatta menzione della loro natura, parlandosi semplicemente di corpi stranieri articolari).

La fig. 1 del lavoro di Moulonguet (35), presa da una Thèse de concours di MorelLavallée (1853), raffigura un caso analogo a quest'ultimo. In essa figura è rappresentato pure un ginocchio aperto, in cui si riconoscono nettamente 4 corpuscoli peduncolati sulla sinoviale; non sono invece riconoscibili lesioni artronosiche a carico della rotula e dei condili femorali.

18 Paul Reichel, nato a Breslavia nel 1858, fu direttore e chirurgo primario all'ospedale civico di Chemnitz dal 1898 al 1930.

19 Al lume di ciò, deve essere considerata come superata la questione di priorità impostata da Wilhelm Müller (38), il quale osservò ed operò, al Luisenhospital di Aachen, un caso di «encondroma diffuso della capsula articolare» a carico della 3 a giuntura metacarpo-falangea sin. di un uomo di 25 anni. Egli comunicò brevemente questo caso il 25 giugno 1898 alla seduta di Düsseldorf della Vereinigung niederrheinisch-westfälischer Chirurgen (36) e lo pubblicò nel $1902(37)$. 
Convinti come siamo dei valori etici ed universali della scienza, siamo ben lungi dal voler dare al presente studio il carattere di una rivendicazione a sfondo sciovinista: tanto più che, come ha detto Virchow $\left({ }^{50}\right)$, «... Morgagni gehört nicht Italien ... allein an; ... so ist er doch ... der Repräsentant der allen Völkern gemeinsamen Wissenschaft geworden».

\section{Luigi Belloni (Milano).}

\section{Bibliographie}

(1) Berry J. - Multiple loose boodies from the knee-joint. Brit.Med. Journ., 1894: I, 1081-81.

(2) Bichat Xavier (1771-1802) - Traité des membranes en général et de diverses membranes en particulier.

Paris (chez Richard, Caille et Ranvier), an VIII (1799).

(3) Bichat X. - Anatomie générale, appliquée à la physiologie et à la médecine. Paris (chez Brosson, Gabon), an X (1801): 2, 142-44.

(4) Bonet Théophile (1620-1689) - Sepulchretum sive anatomia practica, ex cadaveribus morbo denatis, proponens historias et observationes omnium pene humani corporis affectuum, ipsorumque causas reconditas revelans. Genevae (sumptibus Leonardi Chouët), MDCLXXIX.

(5) Bonnet Amédée (1802-1858) - Traité des maladies des articulations. Lyon, $1845: 1,468-89$.

(') Broca Paul (1824-1880) - Nécrose des cartilages. Bull. Soc. Anat. Paris, 1851: 26, 109-09, 165-68, 184-86, 446-49.

() Chiari H. - Chondromatose der Gelenke. Henke-Lubarsch's Hdb. spez. path. Anat. Histol., 1934: 9/II, 79-86.

(8) Cruveilhier Jean (1791-1874) - Essai sur l'anatomie pathologique en général, et sur les transformations et productions organiques en particulier. Paris, 1816: 2, 102-07.

(9) Cruveilhier J. - Anatomie pathologique du corps humain. Paris (J. B. Baillière), 1829-1835: 1, livraison IX.

(10) Fischer I. - Biographisches Lexikon der hervorragenden Ärzte der letzten fünfzig Jahre. Berlin-Wien (Urban \& Schwarzenberg), 1932-1933.

(11) Freund E. - Die Gelenkerkrankung der Bluter. Virch.Arch., 1925: 256, $158-88$.

(12) Freund E. - Über Abstoßungs- und Implantationserscheinungen in erkrankten Gelenken. Virch. Arch., 1927: 263, 515-22.

(13) Giordano D. - Giambattista Morgagni. Torino. (U.T.E.T.), 1941.

(14) Goldschmid E. - Entwicklung und Bibliographie der pathologisch-anatomischen Abbildung. Leipzig (K. W. Hiersemann), 1925.

(15) Gruber Gg. B. - Albrecht von Haller als pathologischer Anatom. Schweiz. med. Wschr., 1938: 68, 828-29. 
(16) Hagemann R. - Gelenkkapselchondrom des Schultergelenks. Med. Klin., 1913: 9, $1243-45$.

(17) von Haller Albrecht (1708-1777) - Opuscula pathologica. Lausannae (Sumpt. Marci-Mic. Bousquet \& Soc.), MDCCLV.

(18) v. Haller A. - Denkschrift herausgegeben von der damit beauftragten Commission auf den 12. December 1877. Bern (B. F. Haller), 1877.

(19) Havers Clopton - Novae quaedam observationes de ossibus et partibus eo pertinentibus: ubi et ratio qua crescunt et nutriuntur exponitur. Versio nova, cui accessit Joannes Ch. Heyne, Lugduni Batavorum (apud Georgium Wishoff), MDCCXXXIV. (La prima edizione dell'opera comparve a Londra nel 1691 sotto il titolo: Osteologia nova or some new observations of the bones and the parts belonging to them, with the manner of the accretion and nutrition; and a discourse of the cartilages in questa opera sono descritti i canali ossei che portano il nome dell'autore.

(20) Hirsch A. - Biographisches Lexikon der hervorragenden Ärzte aller Zeiten und Völker. Berlin-Wien (Urban \& Schwarzenberg), 2. Aufl., 1929-1935.

(21) Humphry G.M. - Loose bodies in the knee-joint. Brit.Med.Journ., 1888: $1,613-13$.

(22) Humphry G. M. - Loose bodies in joints. Brit. Med. Journ., 1888: 2, 707-10.

(23) Kienböck R. - Gelenkosteomatose und Chondromatose. Berlin-Wien (Urban \& Schwarzenberg), 1934.

(24) Knoblich. - Chondroosteomatose. Zbl. Chir., 1928: 55, 1689-90.

(25) König F. - Über freie Körper in den Gelenken. Dtsch. Zschr. Chir., 1888: 27, 90-109.

(26) Kopp J.W. - Osteochondromatose van de Gewrichtskapsel. Nederl. Tijdschr. Geneesk., 1916: 52, 1175-86.

(27) Lannec Réné (1781-1826) - Cartilages accidentels. Dictionaire des Sciences Médicales, 1813: 4, 123-33.

(28) Lang F.J. - Zur Kenntnis der Veränderungen der Hüftpfanne bei Arthritis deformans. Virch. Arch., 1924: 252, 578-664.

(29) Lang F. J. - Arthritis deformans und Spondylitis deformans. Henke-Lubarsch's Hdb. spez. path. Anat. Histol., 1934: 9/II, 252-376.

(30) Lang F.J. - Pathologie der chronischen Gelenkleiden. Dresden-Leipzig (Th. Steinkopff), 1943.

(31) Lexer E. - Gelenkchondrome. Dtsch. Zschr. Chir., 1907: 88, 311-23.

(32) Lotsch F. - Über Chondromatose der Gelenkkapsel. Dtsch. med. Wschr., 1920: $46,544-46$.

(33) Morgagni - Giovanni Battista (1682-1771) - De sedibus et causis morborum per anatomen indagatis libri quinque. Patavii (Sumptibus Remondinianis), MDCCLXV: la prima edizione dell'opera risale al 1761. 
(34) Moritz A.R. - Tabische Arthropathie. (Histologische Studie). Virch. Arch., 1928: $267,746-855$.

(35) Moulonguet $P$. - La signification pathologique des corps étrangers articulaires. Annales d'Anat. Pathol., 1928: 5, 393-439.

${ }^{\left({ }^{36}\right)}$ Müller W. - Demonstrationen zum Kapitel «Fingererkrankungen». (Vereinigung niederrheinisch-westfälischer Chirurgen, I. Sitzung in Düsseldorf am 25. Juni 1898). Dtsch. med.Wschr., 1898: 24 (Vereins-Beil.), 151-51.

(37) Müller W. - Über diffuses Enchondrom der Gelenkkapsel. Arch. klin. Chir., 1902: 66, 637-41.

(38) Müller W. - Discuss. à la communic. faite par Rostock. Zbl. Chir., 1928: 55, 2363-64.

(39) Panner H.J. - Quelques observations sur la chondromatose capsulaire et sur ses caractères radiologiques. Acta Radiol., 1926: 5, 295-303.

(40) Paré Ambroise (1510-1590) - Euvres complètes d'Ambroise Paré . . accompagnées de notes historiques et critiques, et précédées d'une introduction par J.-F. Malgaigne. Paris (J.-B. Ballière), 1841: 3, 32-32.

(41) Pommer Gustav (1851-1935) - Mikroskopische Befunde bei Arthritis deformans. Denkschr. kais. Akad. Wissensch. Wien (Math.-naturwiss. Klasse), 1914: 89, 65-316.

(42) Pommer G. - Über die mikroskopischen Kennzeichen und die Entstehungsbedingungen der Arthritis deformans (nebst neuen Beiträgen zur Kenntnis der Knorpelknötchen). Virch. Arch., 1927: 263, 434-514.

(43) Puccinotti F.P. - Storia della Medicina. Livorno, 1850-1866 (cité par Hirsch).

(44) Reichel P. - Chondromatose der Kniegelenkkapsel. Arch. klin. Chir., 1900: 61, 717-24; Verh.dtsch. Gesell. Chir., 1900: 29/II, 332-339.

(45) von Rokitansky Karl (1804-1878) - Dendritische Vegetationen auf Synovialhäuten. Zschr. Gesell. Ärzte Wien, 1851: 7/1, 2 (cité par Kienböck).

$\left.{ }^{46}\right)$ Rostock P. - Die Gelenkchondromatose. Beitr. klin. Chir., 1928: 144, 58-80.

(47) Tröll A. - Über Gelenkkapselchondrome. Arch. klin. Chir., 1914: 104, 680-93.

(48) Virchow Rudolf (1821-1902) — Die Cellularpathologie in ihrer Begründung auf physiologische und pathologische Gewebelehre. (Zwanzig Vorlesungen, gehalten während der Monate Februar, März und April 1858 im pathologischen Institute zu Berlin). Berlin (A. Hirschwald), 1858.

(49) Virchow R. - Die krankhaften Geschwülste. (Dreißig Vorlesungen, gehalten während des Wintersemesters 1862-1863 an der Universität zu Berlin). Berlin (A. Hirschwald), 1863: 1, 449-62.

(50) Virchow R. - Morgagni und der anatomische Gedanke. Atti XI Congr. Internaz. di Medic., Roma, 1894: 1, 188-97.

(51) Voß I. - Das pathologisch-anatomische Werk Albrecht von Hallers in Göttingen. Göttingen (Vandenhoek und Ruprecht), 1937. 